\title{
Plasma D-Dimer Level in Different Types of Acute Ischaemic Stroke Patients: Experience at a Tertiary Hospital in Dhaka City
}

\author{
Paritosh Kumar Sarkar', Anwar Israil², Mohammad Sayeed Hassan ${ }^{3}$, Abu Nayeem \\ Md. Azharul Hoque ${ }^{5}$ Md. Badrul Alam ${ }^{6}$, Quazi Deen Mohammad \\ ${ }^{1}$ Associate Professor, Department of Neurology, National Institute of Neurosciences \& Hospital, Dhaka, Bangladesh; \\ ${ }^{2}$ Assistant Professor, Department of Neurology, National Institute of Neurosciences \& Hospital, Dhaka, Bangladesh; \\ ${ }^{3}$ Assistant Professor, Department of Neurology, National Institute of Neurosciences \& Hospital, Dhaka, \\ Bangladesh; ${ }^{4}$ Associate Professor, Department of Neurology, National Institute of Neurosciences \& Hospital, \\ Dhaka, Bangladesh; ${ }^{5}$ Professor, Department of Neurology, National Institute of Neurosciences \& Hospital, \\ Dhaka, Bangladesh; ${ }^{6}$ Joint Director \& Professor, Department of Neurology, National Institute of \\ Neurosciences \& Hospital, Dhaka, Bangladesh; ${ }^{7}$ Director \& Professor, Department of Neurology, \\ National Institute of Neurosciences \& Hospital, Dhaka, Bangladesh
}

[Received: 12 October 2018; Accepted: 2 November 2018; Published: 1 January 2019]

\begin{abstract}
Background: Plasma D-Dimer is a biomarker of thrombo-embolism. Objective: The purpose of the present study was to see the plasma D-dimer level in different types of acute ischaemic stroke patients. Methodology: This cross-sectional study was conducted in the Department of Neurology \& Department of Internal Medicine at Dhaka Medical College Hospital, Dhaka, Bangladesh from July 2010 to June 2012 for a period of two (02) years. Patients with ischemic stroke with history within 7 days attending in the stroke clinic of Department of Neurology or admitted in the Department of Neurology and Internal Medicine through the outpatient and emergency Department of Dhaka Medical College Hospital (DMCH) were selected as study population for this study. Analysis of plasma D-Dimer was done in the Department of Hematology of DMCH.b Result: A total of 50 cases were recruited for this study. There were $24.0 \%$ lacunar infarcts, $40.0 \%$ atherothrombotic and $36.0 \%$ embolic infarcts in the study group. Highest level of plasma D-Dimer was observed in embolic $(1700 \pm 964 \eta \mathrm{g} / \mathrm{ml})$ followed by atherothrombotic group $(536 \pm 234 \eta \mathrm{g} / \mathrm{ml})$. The plasma D-Dimer was lowest $(100 \pm 0 \eta \mathrm{g} / \mathrm{ml})$ in lacunar group. Concentration of Plasma D-Dimer showed significant correlation with clinical diagnosis in different subtypes of ischemic stroke $(r=0.902 ; p=0.001)$ and also with risk factors, example, diabetes $(\mathrm{r}=0.319 ; \mathrm{p}=0.012)$ and valvular heart disease $(\mathrm{r}=0.281 ; \mathrm{p}=0.024)$, but no significant correlation with age, hypertension, hyperlipidaemia and smoking. Conclusion: In conclusion plasma D-Dimer is an important bio-marker in the clinical diagnosis and subtypes of ischemic stroke patients. [Journal of National Institute of Neurosciences Bangladesh, 2019;5(1):13-17]
\end{abstract}

Keywords: Plasma D-dimer level; types; acute ischaemic stroke

Correspondence: Dr. Paritosh Kumar Sarkar, Associate Professor, Department of Neurology, National Institute of Neurosciecnes \& Hospital, Sher-E-Bangla Nagar, Agargaon, Dhaka-1207, Bangladesh; Email: sparitosh@hotmail.com; Cell no.: +8801741155177

Conflict of interest: There is no conflict of interest relevant to this paper to disclose.

Funding agency: This research project was not funded by any group or any institution.

Contribution to authors: Sarkar PK \& Israil A have contributed from the protocol preparation, data collection up to report writing. Manuscript writing was performed by Hassan MS, Nayeem A \& Hoque MA. Statistical analysis was performed by Hassan MS. Alam MB \& Mohammad QD have involved in supervision of the research work and revision of manuscript.

How to cite this article: Sarkar PK, Israil A, Hassan MS, Nayeem A, Hoque MA, Alam MB, Mohammad QD. Plasma D-Dimer Level in Different Types of Acute Ischaemic Stroke Patients: Experience at a Tertiary Hospital in Dhaka City. J Natl Inst Neurosci Bangladesh, 2019;5(1): 13-17

Copyright: (C2019. Sarkar et al. Published by Journal of National Institute of Neurosciences Bangladesh. This article is published under the Creative Commons CC BY-NC License (https://creativecommons.org/licenses/by-nc/4.0/). This license permits use, distribution and reproduction in any medium, provided the original work is properly cited, and is not used for commercial purposes.

\section{Introduction}

Stroke is the leading cause of mortality and morbidity worldwide ${ }^{1}$. Globally stroke is the second leading cause of death above the age of 60 years and the fifth leading cause in people aged 15 to 59 years of age ${ }^{2}$. In many developed countries the incidence of stroke declining but the actual number is increasing because of ageing populations. Bangladesh is a developing country with 150 million population of which one third is in older age group ${ }^{1}$. In a survey conducted in Dhaka city and some parts of Bangladesh revealed crude prevalence of stroke is 3 per 1000 population ${ }^{3}$. 
Plasma level of D-Dimer is an index of fibrin generation, which is a cross linked fibrin degraded products. Moreover, tissue plasminogen activator is needed for formation of plasmin from plasminogen. Several studies show that substantial alternations in hemostatic function in there cohort of patients with cerebral ischemia ${ }^{4}$. These consist of sustained increase in platelet activation, increased thrombin activity acutely, and decrease in fibrinolytic activity. D-Dimer levels were significantly elevated in patients with acute ischemic stroke, indicating increased fibrin formation and it is consistent with the concept that an occlusive fibrin thrombus, either embolic or form in situ, is involved in the pathogenesis of ischemic stroke. D-Dimer levels is highest in the subjects with cardioembolic stroke, less high in patients with large vessel disease and not elevated in lacunar stroke or $\mathrm{TIA}^{5}$. These findings suggest that plasma D-Dimer levels correlate with the size of the fibrin thrombus, and thus patients with lacunar disease and TIA either have thrombi too small to produce detectableelevations of plasma D-Dimer, or that fibrin formation does not occur to significant extent. In this context, this present study was undertaken to see the plasma D-dimer level in different types of acute ischaemic stroke patients.

\section{Methodology}

This study was designed as analytical cross-sectional study. This study was conducted in the Department of Neurology \& Department of Internal Medicine at Dhaka Medical College Hospital, Dhaka, Bangladesh from July 2010 to June 2012 for a period of two (02) years. Patients with ischemic stroke with history within 7 days attending in the stroke clinic of Department of Neurology or admitted in the Department of Neurology and Internal Medicine through the outpatient and emergency Department of Dhaka Medical College Hospital (DMCH) were selected as study population for this study. The selection of the study was performed by purposive sampling technique. Patients admitted with signs and symptoms of acute ischemic stroke, patients diagnosed as a case of acute ischemic stroke by CT scan of brain were included for this study. Patients below 18 years of age, concomitant use of anticoagulant in stroke patient and patients with co-morbidity that affects D-Dimer level were excluded from this study. Ischemic stroke patients fulfilling the inclusion and exclusion criteria were enrolled for the study, examined by the investigator. Clinical diagnosis of stroke was done according to National Institute of Neurological Disorders and Stroke (NINDS) criteria6. Then CT scan was done to confirm the diagnosis. Analysis of plasma
D-Dimer was done in the Department of Hematology of $\mathrm{DMCH}$. Commercially prepared reagent 'CTM D-Dimer Latex Kit' was used for analysis. CTM D-Dimer latex test is intended for the rapid qualitative or semi quantitative evaluation of circulating derivatives of cross linked fibrin degradation products (XL-FDP) in human plasma. CTM D-Dimer latex is a rapid agglutination assay utilizing latex beads coupled with a highly specific D-Dimer monoclonal antibody. Undiluted plasma D-Dimer concentration was regarded as Positive or Negative depending on plasma value, either greater or less $200 \eta \mathrm{g} / \mathrm{ml}$ respectively. Quantitative measurement was done by serial dilution method. All collected data were related with clinical history, physical examination, biochemical and radiological findings with Serum D-Dimer was analyzed. Statistical analysis was performed with the help of computer by using statistical package for social science (SPSS). All qualitative data were expressed in frequency and percentage. ANOVA test was performed among the mean with SD among the subtypes of ischaemic stroke. The levels of significance accepted at $P$ value $\leq 0.05$. Permission from the Ethical Committee was taken before conducting the study.

\section{Results}

A total of 50 cases were recruited for this study of which $16 \%$ patients were from 18 to 40 years age group. The maximum number of patients was found in more than 60 years of age group which is $32 \%$. The mean $( \pm \mathrm{SD})$ of ages were $58.36 \pm 14.80$ in the ischemic stroke (Table 1).

Table 1: Distribution of age in the study groups

\begin{tabular}{|c|c|c|}
\hline Age Group & Frequency & Percentage \\
\hline 18 to 40 Years & 8 & 16.0 \\
\hline 41 to 50 Years & 11 & 22.0 \\
\hline 51 to 60 Years & 7 & 14.0 \\
\hline More than 60 Years & 24 & 48.0 \\
\hline Total & 50 & 100.0 \\
\hline
\end{tabular}

Etiological subtypes of acute ischemic stroke among study population were recorded. There were $24.0 \%$ lacunar infarcts, $40.0 \%$ atherothrombotic and $36.0 \%$ embolic infarcts in the study group (Table 2).

The mean plasma concentration in different subtypes of ischemic stroke were measured. Mean plasma D-Dimer level was highest in embolic infarct $(1700 \pm 964 \eta \mathrm{g} / \mathrm{ml})$ followed by atherothrombotic $(536 \pm 234 \eta \mathrm{g} / \mathrm{ml})$ and lowest in lacunar $(100 \pm 00 \eta \mathrm{g} / \mathrm{ml})$ subtype. A 
significant difference $(\mathrm{F}=31.16 ; \mathrm{p}=0.001)$ observed in the mean plasma D-Dimer level in the subtypes of stroke.

Table 2: Etiological Subtypes of Acute Ischemic Stroke among Study Population

\begin{tabular}{lcc}
\hline Subtypes & Frequency & Percentage \\
\hline Lacunar & 12 & 24.0 \\
Atherothrombotic & 20 & 40.0 \\
Embolic & 18 & 36.0 \\
\hline
\end{tabular}

Table 3: Plasma D-Dimer Level in Etiological Subtypes of Acute Ischemic Stroke ( $\eta \mathrm{g} / \mathrm{ml})$

\begin{tabular}{lcc}
\hline Subtypes & Mean \pm SD & p value \\
\hline Lacunar & $100.0 \pm 00$ & 0.001 \\
Atherothrombotic & $536.0 \pm 234$ & \\
Embolic & $1700.0 \pm 964$ & \\
Total & $\mathbf{8 0 4} \pm \mathbf{1 2 0}$ & \\
\hline
\end{tabular}

One way ANOVA was performed to see the level of significant.

The plot of mean among the subtype of stroke was projected in figure I.

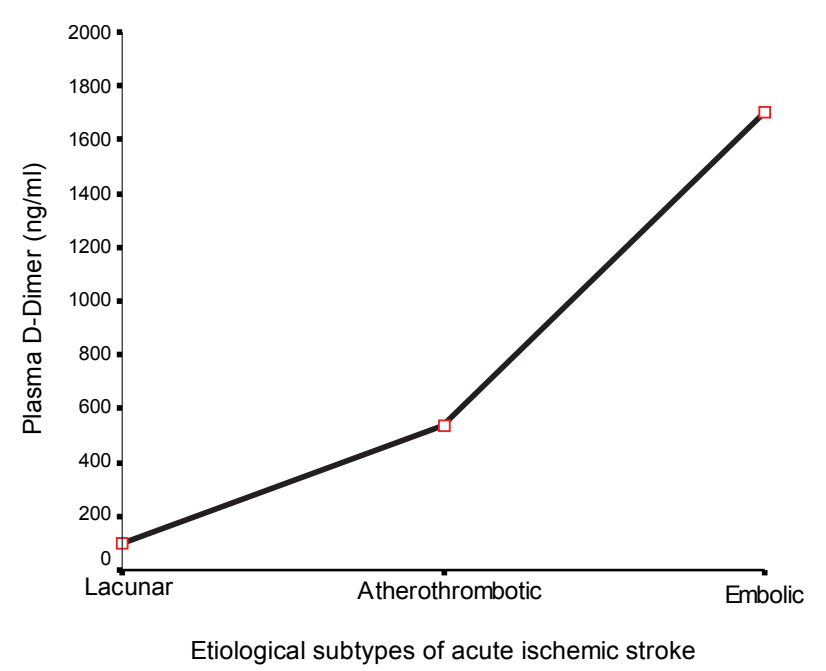

Figure I: Quantitative assay of Plasma D-Dimer in etiological subtypes of acute ischemic stroke

The correlation of plasma D-Dimer with different independent variables of ischemic stroke were measured among the study population. Concentration of Plasma D-Dimer showed significant correlation with clinical diagnosis in different subtypes of ischemic stroke $(\mathrm{r}=0.902 ; \mathrm{p}=0.001)$ and also with risk factors, example, diabetes $(\mathrm{r}=0.319 ; \mathrm{p}=0.012)$ and valvular heart disease $(\mathrm{r}=0.281 ; \mathrm{p}=0.024)$, but no significant correlation with age, hypertension, hyperlipidaemia and smoking.
Table 4: Correlations of Plasma D-Dimer with different variables in the studied subjects

\begin{tabular}{lcc}
\hline Determinants of 'r' & \multicolumn{2}{c}{ Ischemic Stroke Group } \\
\cline { 2 - 3 } & $\mathbf{r}$ & P value \\
\hline Plasma D-Dimer vs & $\mathbf{0 . 9 0 2}$ & $\mathbf{0 . 0 0 1}$ \\
Ischemic stroke subtypes & & \\
\hline
\end{tabular}

\section{Discussion}

Diagnosis of stroke is still challenging and is mostly based on clinical findings ${ }^{2}$. Patients developed a sudden onset of focal neurological deficit may have a stroke or may have one of several possible 'stroke mimic' conditions including the aura of migraine, postictal deficits following a focal seizures, metabolic derangements (hypoglycaemia), an anamnestic spell (recrudescence of prior deficit in the setting of an infection or metabolic derangement), mass lesion or a conversion reaction ${ }^{4}$.

The most of the biomarkers of stroke are panel biomarker and studies correlating there plasma concentration to stroke phenotypes has not been established. Some of these are found to be increased in the cerebrospinal fluid of stroke patients but serum concentrations do not differ from those control patients ${ }^{7}$. The ideal biomarker would clearly distinguish stroke from stroke mimics and have consistent results within and between laboratories. It would be a blood or serum or plasma test and would be diagnostic within the first few hour of the onset of stroke with a quick automated assay ${ }^{8}$.

Stroke biomarkers most useful when combined with clinical judgment alone or with clinical judgment plus brain imaging9. Fibrin D-Dimer could be an ideal hemostatic marker for early diagnosis of stroke in this ground. Raised plasma D-Dimer is a constant finding in thrombogenic disorder, especially when they affect the venous system. Several authors reported that the increased level of D-Dimer always related to the acute phase of ischemic stroke and related this to worse vital and functional prognosis and to a higher risk of recurrence during follow up ${ }^{10}$. Study conducted by Barber et $\mathrm{al}^{11}$ have concluded that plasma D-Dimer is an independent marker of acute progressing stroke. It is may be positive as early as 30 minutes of stroke and remains positive up to one month. D-Dimer level could be estimated from the blood samples which were collected within 7 days of stroke for assessing stroke progression. Estimation of D-Dimer may also play role in differentiating stroke subtypes ${ }^{12}$. There are other several conditions associated with increased D-Dimer, 
such as pregnancy, after surgery, trauma, pre-eclampsia, malignancy, infection, disseminated intravascular coaglution (DIC), sickle cell disease, pulmonary thromboembolism, deep vein thrombosis (DVT) and acute coronary syndrome ${ }^{13}$.

Plasma D-Dimer level was measured in 50 acute ischemic stroke patients and was compared with the age and sex matched healthy control groups. The results of the present study demonstrate that D-Dimer level significantly differ among the stroke subtypes after an acute ischemic event and the measurement of D-Dimer levels can be reliable in the early diagnosis of the mechanism of underlying cause. In a series of 291 consecutive stroke patients in Bangladesh revealed mean age was 57.9 years and incidence of stroke was $80.0 \%$ in male patients ${ }^{14}$. This study reveals mean age of stroke as $58.36 \pm 14.80$ years.

Ischemic stroke has been classified according to the Oxfordshire community ${ }^{15}$ stroke project criteria based on clinical symptoms, location, and extent of cerebral infarction and sub divided into 4 clinical categories, total anterior circulation infracts (TACI), partial anterior circulation infarcts (PACI), lacunar infarcts (LACI), and posterior circulation infarcts (POCI). Several studies have found that an increased concentration of plasma D-Dimer level in acute ischemic stroke patients ${ }^{16-17}$. This D-Dimer value showed weak to moderate correlation, supports a link between inflammation, hypercoagubility and fibrin turnover in ischemic stroke patients ${ }^{16}$. This present study reveals that mean concentration of plasma D-Dimer is $804 \pm 120 \eta \mathrm{g} / \mathrm{ml}$ in all ischemic stroke patients irrespective of subtypes and a value $126 \pm 16 \eta$ $\mathrm{g} / \mathrm{ml}$ in control group. Furthermore, the plasma

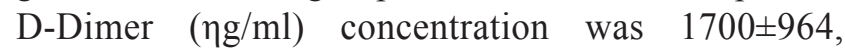
$536 \pm 234$ and $100 \pm 0$ in embolic, atherothrombotic and lacunar group respectively. Values of plasma D-Dimer varied significantly in different subtypes of stroke in this study although lowest concentration in lacunar group is contrary to the one study ${ }^{18}$.

Plasma D-Dimer value is identified in patients at high risk for stroke progression and remain as an independent predictor ${ }^{11}$. Raised D-Dimer is a constant finding in thrombogenic disorders. In a study by Barber and his colleagues in 2004 reported increased levels of D-Dimer during the acute phase of ischemic stroke and related to this worse vital function and functional prognosis and to a higher risk of recurrence during follow-up ${ }^{11}$. In addition, plasma D-Dimer value is highest in association with cardioembolic mechanism. A controlled study reported that patients with cardioembolic stroke had raised mean levels of D-Dimer, and has higher risk of new embolisms ${ }^{19}$. Alvarez-Perez et $\mathrm{al}^{10}$ concluded in a study that there was a continuous activation of coagulation and fibrinolysis characterized by raised D-Dimer in ischemic stroke. This study is taken for early identification of acute ischemic stroke patients in Bangladeshi population, thus stroke patients may be benefited with thrombolytic therapy with reduction of substantial morbidity and mortality.

This study suggests that plasma D-Dimer level is a potential biomarker in acute ischemic stroke and also helpful in categorizing the subtypes. Estimation of plasma D-Dimer level and adjunct to CT scan will strengthen the diagnosis of acute ischemic stroke which will help in therapeutic management.

Present study has some limitations. A small number of cases were included in this study. Follow up was not done. Stroke progression was not evaluated with further measurement of plasma D-Dimer level. A prospective randomized study with greater number of patients will help to ascertain further role of plasma D-Dimer in acute ischemic stroke.

\section{Conclusion}

This study indicates that raised plasma D-Dimer is a constant finding in acute phase of ischemic stroke. Measurement of plasma D-Dimer is simple and can be an adjunct to diagnosis of acute ischemic stroke. Thus measuring plasma D-Dimer level will further strengthen the clinical diagnosis of ischemic stroke particularly where CT scan could not be done. Plasma D-Dimer estimation should be done in acute ischemic stroke patients, especially in embolic and atherothrombotic subgroups for thrombolysis and interventions.

\section{References}

1. Strong K, Mathers C, Bonita R. Preventing Stroke: Saving lives around the world. Lancet Neurol. 2007; 6(2): 182-187

2. World Health Organization. The World Health Report 2004: Changing history. Geneva: WHO Bull, 2004.

3. Mohammad QD, Habib M, Hoque MA, Mondol MBA, Haque B, Hossain S, Rahman KM and Khan SU. Prevalence of stroke in a Bangladeshi population aged forty years and above. Mymensingh Medical Journal. 2011; 20(4): 640-644

4. Fisher $M$ and Francis R. Altered coagulation in cerebral ischemia, platelet, thrombin and plasmin activity. Arch Neurol. 1990; 47: 1075-1079

5. Cote R, Wolfson C, Solymoss S, Mackey A, Leclerc JR, Simard D, Rouah F, Bourque F and Leger B. Hemostatic Markers in Patients at Risk of Cerebral Ischemia. Stroke. 2000; 31: 1856-1862 6. Kidwell SC and Warach S. Acute ischemic Cerebrovascular Syndrome: Diagnostic criteria. Stroke. 2003; 34: 2995-2998. 
7. Reynolds MA Howard J, Kirchick, Dahlen JR et al., Early biomarkers of stroke. Clinical chemistry. 2003; 49(10): 1733-1739 8. Jensen MB, Chacon MR, Sattin JA, Levine RL and Vemuganti R. Potential biomarkers for the diagnosis of stroke. Cardiovasc Ther. 2009; 7(4): 389-393

9. Whiteley W, Tseng MC, Sandercock P. Blood biomarkers in the diagnosis of ischemic stroke: a systematic review. Stroke. 2008; 39: 2902-2909

10. Alvarez-Perez FG, Castelo-Branco M, Alvarez-Sabin. Usefulness of measurement of fibrinogen, D-Dimer, D-dimer/fibrinogen ratio, C-reactive protein and erythrocytes sedimentation rate to assess the pathophysiology and mechanism of ischemic stroke. J Neurol Neursurg Psychiarty. 2011

11. Barber M, Langhorne P, Rumley A, Lowe DG, Stott DJ. Haemostatic Function and progressing ischemic stroke: D-Dimer predicts Early clinical progression. Stroke. 2004; 35:1421-1430

12. Feinberg MW, Erickson PL, Bruck D, Kittelson J. Hemostatic Marker in Acute Ischemic Stroke. Association with stroke subtypes, severity and outcome. Stroke. 1996; 27: 1296-1300 13. Wakai A, Glesson A, Winter D. Role of fibrin D-Dimer testing in emergency medicine. Emerg Med J. 2003; 20:310-325
14. Chowdhury AH, Yokoyama T, Kokubo Y, Zaman MM, Haque A and Tanaka H. Apolipoprotein E Genetic Polymorphism and Stroke Subtypes in a Bangladeshi Hospital-Based Study. Journal of Epidemiology. 2001; 11: 131-138

15. Tei H, Uchiyama S, Ohara $\mathrm{K}$, et al., Deteriorating Ischemic Stroke in 4 Clinical Categories Classified by the Oxfordshire Community Stroke Project. Stroke. 2000; 31: 2049-2054

16. Napoli MD and Papa F. Inflammation, Hemostatic Markers, and Antithrombotic Agents in Relation to Long-Term Risk of New Cardiovascular Events in First-Ever Ischemic Stroke. Stroke. 2002; 33: 1763-1771

17. Hankay G and Warlow C. Treatment and secondary prevention of stroke: evidence, costs, and effects on individuals and populations. Lancet. 1999; 354: 1457-1463

18. Montaner J, Perea-Gainza M, Delgado P, Ribo M, Chacon P, Rosell A, Quintana M, Palacios ME, Molina CA, Alvarez-Sabin J. Etiologic diagnosis of ischemic stroke subtypes with plasma biomarkers. Stroke. 2008; 39(8):2280-2287

19. Lip GYH, Lowe GDO, Rumley A et al. Increased marker of thrombogenesis in chronic atrial fibrillation: effects of warferin treatment. Br Heart J. 1995; 73:527-533 